\title{
Comparisons of Precipitation Measurements by the Advanced Microwave Precipitation Radiometer and Multiparameter Radar
}

\author{
J. Vivekanandan, Joseph Turk, Associate Member, IEEE, and V. N. Bringi
}

\begin{abstract}
Multiparameter microwave radar measurements are based on dual-polarization and dual-frequency techniques and are well suited for microphysical inferences of complex precipitating clouds, since they depend upon the size, shape, composition, and orientation of a collection of discrete random scatterers. Passive microwave radiometer observations represent path integrated scattering and absorption phenomena of the same scatterers. The response of the upwelling brightness temperatures $T_{B}$ to the precipitation structure depends on the vertical distribution of the various hydrometeors and gases, and the surface features. As a result, combinations of both active and passive techniques contain great potential to markedly improve the longstanding issue of precipitation measurement from space.

The NASA airborne Advanced Microwave Precipitation Radiometer (AMPR) and the National Center for Atmospheric Research (NCAR) CP-2 multiparameter radar were jointly operated during the 1991 Convection and Precipitation/Electrification experiment (CaPE) in central Florida. The AMPR is a four channel, high resolution, across-track scanning total power radiometer system using the identical multifrequency feedhorn as the widely utilized Special Sensor Microwave/Imager (SSM/I) satellite system. Surface and precipitation features are separable based on the $T_{B}$ behavior as a function of the AMPR channels. The radar observations are presented in a remapped format suitable for comparison with the multifrequency AMPR imagery. Striking resemblances are noted between the AMPR imagery and the radar reflectivity at successive heights, while vertical profiles of the CP-2 products along the nadir trace suggest a storm structure consistent with the viewed AMPR $T_{B}$. Directly over the storm cores, the difference between the 37 and $85 \mathrm{GHz} T_{B}$ was noted to approach (and in some cases fall below) zero. Microwave radiative transfer computations show that this is theoretically possible for hail regions suspended aloft in the core of strong convective storms.
\end{abstract}

\section{INTRODUCTION}

$\mathbf{T}$ HE spaceborne measurement and monitoring of rainfall is a topic of major interest since it influences the global hydrologic cycle and the nature of climate variations. In addition, newly proposed Earth-space satellite communications will utilize Ka-band frequencies $(18-30 \mathrm{GHz})$, where in addition to water vapor and clouds, attenuation and depolarization

Manuscript received August 18, 1992; revised March 1, 1993. This work was supported by the NASA Earth Science and Applications Division under Grant NAG8-890. The National Center for Atmospheric Research is sponsored by the National Science Foundation.

J. Vivekanandan is with the Research Applications Program, National Center for Atmospheric Research, Boulder, CO 80307.

J. Turk and V. N. Bringi are with the Department of Electrical Engineering, Colorado State University, Fort Collins, CO 80523.

IEEE Log Number 9209087. effects due to rain, melting, and ice cannot be neglected. Combinations of both radar and passive microwave sensors are being investigated in projects such as the Tropical Rainfall Measuring Mission (TRMM) [1]. Microwave Doppler radar is capable of mapping the vertical profiles of cloud parameters such as cloud top heights, ice extent, melting region, and rainfall intensity, which are decisive factors in determining the upwelling equivalent blackbody brightness temperature $T_{B}$ measured by a passive spaceborne system, or the path attenuation suffered along Earth-space communication links.

Multiparameter radar measurements are based on dualpolarization and dual-frequency techniques, and can yield significantly more microphysical information such as mean shape, size, and orientation of an ensemble of scatterers. A modern multiparameter radar is capable of measuring both range-resolved backscatter parameters such as reflectivity $Z$, differential reflectivity $Z_{D R}$, linear depolarization ratio $L D R$, and the zero-lag cross correlation $\rho_{H V}(0)$ ), as well as the range cumulative differential propagation phase $\phi_{D P}$. For dual-wavelength radars operating at $S$ band $(3 \mathrm{GHz})$ and $X$ band $(10 \mathrm{GHz})$, the cumulative attenuation is also measurable, assuming negligible propagation effects at $S$ band. Both the specific differential propagation phase $K_{D P}$ and the specific attenuation rate $A$ are the range derivatives of $\phi_{D P}$ and the cumulative attenuation, respectively. After correcting the $X$ band returns for propagation attenuation, the resulting difference between the $S$ and $X$ band reflectivities is known as the dual-frequency ratio hail signal (DFR-HS) [2]. At $X$ band, hail behaves as a Mie scatterer, producing a lower measured reflectivity than at $S$ band [3], [4]. For applications related to microwave radiative transfer, approximate locations of the ice, melting, and rain layers can be estimated using these multiparameter observables. In particular, the $X$ band specific attenuation rate (denoted by $A_{X}$ ) and $S$ band specific differential propagation phase $\left(K_{D P}\right)$ are closely related to rain water content [5]. $Z_{D R}$ has been shown to be useful for differentiating between rain and ice [6]. Hail detection using $Z_{D R}$ and DFR-HS is documented [7]. At 10- $\mathrm{cm}$ wavelength, $Z_{D R}$ is an estimator of the reflectivity weighted mean axis ratio of the raindrops filling the radar resolution volume [8]. Depolarization of the radar echo which is quantified by the linear depolarization ratio $L D R$ can be used to determine the melting layer width [9]. The $X$ band specific attenuation is approximately related to the fourth moment of the rain drop size distribution. The above mentioned multiparameter radar 
observables show marked differences in the ice, melting ice, and rain regions because the shape, orientation, and dielectric constant are distinctly different in each region. Near and below the $0^{\circ} \mathrm{C}$ level, the radar pulse volume is often filled with mixed phases of water and ice. Depressions in the zero-lag cross correlation $\rho_{H V}(0)$ occur in mixed phase precipitation [10]. In principle, using the polarimetric measurable triplet $\left(Z_{H}, Z_{D R}\right.$ and $\left.K_{D P}\right)$, the individual rain and hail precipitation rates can be estimated in a rain-hail mixed media [11]. Fulton and Heymsfield [12] tied together the evolution of the CP2 multiparameter radar products with a series of aircraft microwave $T_{B}$ measurements between 18 and $183 \mathrm{GHz}$.

However, for global precipitation retrieval, especially over the ocean where scant rainfall observations are available, it is likely that polar orbiting satellite-based passive microwave radiometers will remain the most widely used instrumentation for some years to come. Since 1987, the Defense Meteorological Systems Package (DMSP) satellites have carried into polar orbit several of the Special Sensor Microwave/Imager (SSM/I) multifrequency microwave radiometers. It is fair to say that this radiometer package has been well received and finds widespread geophysical applications. For the case of precipitation retrieval, the response of the upwelling brightness temperatures $T_{B}$ to the precipitation structure depends upon the spatial distribution of the various hydrometeors, gases, and the surface characteristics within the radiometer field of view. In the $10-100 \mathrm{GHz}$ range, small cloud water drops are absorbers (albedo $\approx 0$ ), rain is a fairly good absorber (albedo $\approx 0.3-0.5$ ), and ice is nearly nonabsorbing (albedo $\approx 0.60-0.99$ depending on frequency and density). It is well known that, in general, the lower passive microwave frequencies-the emission-based frequencies below $20 \mathrm{GHz}$-are most sensitive to processes originating at lower altitudes in the cloud, while the scattering-based frequencies above 35 $\mathrm{GHz}$ respond to the higher altitudes near and above the $0^{\circ} \mathrm{C}$ level [13]-[15]. At these frequencies, upwelling thermal emission is scattered away from the radiometer field of view (FOV) by the presence of high albedo graupel or snow, while emission-based methods are generally made below $20 \mathrm{GHz}$ and are primarily utilized for the estimation of rainfall over the ocean. However, such categorization can be misleading since in actuality, nonprecipitating cloud water is often spread throughout the cloud [16], and even at $19 \mathrm{GHz}$, scattering effects from graupel can be significant [17].

Rainfall estimation over an ocean background has been studied more extensively than land-based rainfall. Low ocean surface emissivity results in a radiometrically cold background, where emission/absorption processes within any cloud water or moderate rain produces an increase in the brightness temperature. This is essentially the idea behind oceanic $19 \mathrm{GHz} T_{B}-R$ relations for estimation of rain rates less than $20 \mathrm{~mm} / \mathrm{h}^{-1}$ over the ocean [18]. Also, emission from most ocean surfaces exhibits a substantial polarization difference between its $V$ and $\mathrm{H} T_{B}$ at scan angles away from nadir. The surface polarization differences are overcome due to unpolarized radiation emitted by precipitation. Thus precipitation intensity can be related to the decrease in polarization differences [13]. However, the above described simplistic radiation transfer process is complicated by the presence of melting, cloud water, and highly variable and unestablished parameters of ice particles, such as average and maximum size and the corresponding density [19]-[21].

Over land, rainfall estimation is restricted by the lack of $T_{B}$ contrast between land and precipitation. Theoretical studies using radar and a radiative transfer model have shown that the $T_{B}$ response above $37 \mathrm{GHz}$ is relatively insensitive to the rain mass content below, being controlled by the optical thickness of the overlying ice region [20]. Although there is certainly a physical process that connects the ice and rain phase intensities, there is also a time lag associated with the fallout of ice particles into rain. As a result, precipitation rate estimation from 37 and $85 \mathrm{GHz}$ scattering channels display a large scatter in their behavior [19], and depend rather widely upon the evolutionary stage of the cloud system. In general, $T_{B}$ observations over precipitation from 37 to $85 \mathrm{GHz}$ are sensitive to both large ice particles and supercooled cloud drops, the latter of which can "mask" the ice scattering [19].

Recently, there have been a number of radiative transfer models developed which incorporate multifrequency, multipolarization components to facilitate the understanding of a variety of observed brightness temperatures. These are vertically and angularly detailed plane-parallel radiative transfer models [22], [23]. It is fair to state that most of the modeling studies emphasize that the improved characterization of vertical structure of precipitation (ice, melting, and rain layer) will require multifrequency passive observations. Based on the current state of understanding of the interaction between microwaves and the rainfall process, there is ample scope for improvement, testing, and verification of remote sensing techniques by combining both active and passive microwave methods. In this investigation, NCAR CP-2 multiparameter radar observations from the 1991 Convection and Precipitation/Electrification experiment (CaPE) [24] in central Florida are presented. The CaPE experiment provided the premier opportunity to gather concurrent measurements with the NASA Advanced Microwave Precipitation Radiometer (AMPR) and a multiparameter radar. The AMPR is a four channel, across-track scanning radiometer package which was mounted aboard a NASA ER-2 high altitude aircraft. The high resolution AMPR $T_{B}$ imagery is presented against the CP-2 radar imagery. Behavior of the multifrequency $T_{B}$ is explained in terms of the various radar products, which were remapped to facilitate both visual and quantitative comparisons of the AMPR imagery. Current investigations are focused upon testing and verification of a multispectral cloud-modelbased precipitation retrieval algorithm [25], and improving the inversion of multiparameter radar products into physical quantities.

\section{RADAR AND RADIOMETER INSTRUMENTATION}

The instrumentation configuration is noteworthy in several respects. First, the latter three of the AMPR channels correspond to frequencies used with the SSM/I system. Second, the upcoming TRMM experiment will involve a spaceborne 14$\mathrm{GHz}$ radar along with a full complement of microwave and 
infrared passive sensors, including a $10-\mathrm{GHz}$ channel. Third, as the cost, size, and power consumption of millimeter wave devices continues to decrease, it is possible that microwave channels may be included on future geostationary satellite systems. The multifrequency high-resolution imagery available from the AMPR provides the necessary observational platform with which to gauge the past, current, and future requirements for passive microwave radiometry from space.

\section{A. The CP-2 Multiparameter Radar}

The CP-2 multiparameter radar is operated and maintained by the National Center for Atmospheric Research (NCAR), in Boulder, CO. It is primarily designed for cloud physics research. The CP-2 radar operates in a linear polarization basis at both the $S(3 \mathrm{GHz})$ and $X(10 \mathrm{GHz})$ frequency bands [26]. In addition to measuring the usual horizontallypolarized copolar reflectivity at $S$ band, a high-power ferrite circulator switch is used to alternate the receive and transmit polarization states between horizontal $(\mathrm{H})$ and vertical $(\mathrm{V})$ with a PRT of approximately $1 \mathrm{~ms}$. This arrangement enables the measurement of the $S$ band $Z_{D R}$. With a coherent receiver it also measures the conventional mean Doppler velocity and spectrum width with an unambiguous velocity of $13 \mathrm{~m} \mathrm{~s}^{-1}$. Typically 64 or $128 \mathrm{H}$ and V samples are used with 512 range gates spaced 200 or $300 \mathrm{~m}$ apart, giving a maximum range of $100-150 \mathrm{~km}$. For the data described here, the differential phase $\phi_{D P}$ and zero-lag cross correlation $\rho_{H V}(0)$ [5] were not collected by CP- 2 . The antenna assembly consists of a dual-mode Potter horn supported at the focus of an 8.5$m$ parabolic reflector, with a resultant antenna beamwidth of $0.93^{\circ}$. Antenna scan rate is $\approx 6^{\circ} \mathrm{s}^{-1}$ in azimuth, and it typically requires under five minutes to obtain a sector scan of the cloud area under investigation.

At $X$ band, two antennas are used, one for $\mathrm{H}$ transmit and copolar reception, and the other for reception of the crosspolar $\mathrm{V}$ return. Both receivers are incoherent. The $X$ band system sits atop the $S$ band reflector and has two identical polarization-twist Cassegrain antennas which are mounted orthogonal to each other. Using this dual-channel receiver system, the $X$ band system can measure reflectivity and $L D R$. Both the $X$ band system and $S$ band antenna are mounted on the same pedestal and they have matched beamwidths. Thus the CP-2 radar, with its dual-frequency and dual-polarization capabilities, offers a diverse combination of measurements for investigating both cloud dynamics and microphysics.

\section{B. The Advanced Microwave Precipitation Radiometer (AMPR)}

The Advanced Microwave Precipitation Radiometer (AMPR) was designed and built in 1990 by the Georgia Tech Research Institute under the auspices of the NASA Marshall Space Flight Center (MSFC). The AMPR is constructed specifically to mount within the lower bay of a NASA ER-2 high altitude aircraft [27]. It utilizes the identical multifrequency feedhorn (MFFH) as the current DMSP Special Sensor Microwave/Imager (SSM/I) for its $19.35,37.1$, and $85.5 \mathrm{GHz}$ channels, with a separate feedhorn for a $10.7-\mathrm{GHz}$ channel. A flat reflective plate is used to direct the upwelling emission from the scene through a dielectric lens and into each of the respective feedhorns. The $3-\mathrm{dB}$ beamwidths range from $8^{\circ}$ at $10.7 \mathrm{GHz}$ to $1.8^{\circ}$ at $85.5 \mathrm{GHz}$, providing nadir resolutions on the Earth's surface of $2.8 \mathrm{~km}$ at 10.7 and $19.35 \mathrm{GHz}, 1.5$ $\mathrm{km}$ at $37.1 \mathrm{GHz}$, and $640 \mathrm{~m}$ at $85.5 \mathrm{GHz}$. A microprocessorcontrolled stepper motor spins the reflector such that the observed scene is scanned across-track, starting at $+45^{\circ}$ from nadir (pilot's right), through nadir and finishing at $-45^{\circ}$ (pilot's left). Each scan requires $3 \mathrm{~s}$, during which time the aircraft moves forward about $700 \mathrm{~m}$. The reflector then moves back to $+45^{\circ}$ and the process is repeated. After every four scene scans, the reflector rotates upward to stare at hot and cold calibration loads for $3 \mathrm{~s}$ total. The scan rate is sufficient to provide contiguous mapping at $85.5 \mathrm{GHz}$, with spatial oversampling occurring for the three lower frequencies. The receivers are total power designs with an integration time of $50 \mathrm{~ms}$, providing time for 50 integrator samples (image pixels) across-track. For an ER-2 flight altitude of $20 \mathrm{~km}$, the equivalent across-track swath width on Earth is $\approx 40 \mathrm{~km}$. The ER-2 airspeed is $\approx 13 \mathrm{~km} / \mathrm{min}^{-1}$.

Unlike the SSM/I, the AMPR currently uses a single MFFH port for its three highest channels, with this and the $10-\mathrm{GHz}$ feedhorn mounted such that the receive polarization state at nadir is linear $45^{\circ}$. Consequently, the receive polarization state is always linear, changing from fully horizontal polarization (H) at the scan start to fully vertical polarization (V) at the scan end. The end result is a polarization mixing across the scan. As discussed by [28], a rotating polarization basis of the instrument causes a skew of the instrument's polarization basis with respect to the scene's polarization basis. With the current AMPR feedhorn configuration and a single receiver per frequency, it provides true scene $\mathrm{H}$ and $\mathrm{V} T_{B}$ only at each of its scan limits. However, its polarization state always remains a linear combination of the scene $\mathrm{H}$ and $\mathrm{V}$ across its scan according to

$$
T_{B}(\theta)=T_{B}^{H} \cos ^{2}\left(\theta-45^{\circ}\right)+T_{B}^{V} \sin ^{2}\left(\theta-45^{\circ}\right)
$$

where $\theta$ ranges from $+45^{\circ}$ at the scan start to $-45^{\circ}$ at the scan end, and $T_{B}^{H}$ and $T_{B}^{V}$ are the $\mathrm{H}$ and $\mathrm{V} T_{B}$ in the scene polarization basis. Equation (1) was verified by selecting an AMPR scan which took place over a calm oceanic noncloudy region. For such conditions, the $10-\mathrm{GHz}$ channel is the least affected by water vapor, and hence only mildly obscures the polarized ocean $T_{B}$. For radiative transfer applications, the simplest ocean model is that of a specular plane surface. Its emissivity (and hence brightness temperature) increases as a function of scan angle from nadir when receiving $\mathrm{V}$ polarized scene emission, and decreases in the case of $\mathrm{H}$ polarized scene emission. At nadir, specular $\mathrm{H}$ and $\mathrm{V}$ ocean emissivities are equal and range from 0.35 at $10 \mathrm{GHz}$ to 0.6 at $85 \mathrm{GHz}$. Fig. 1 shows a single ocean scan near 223400 UTC on 12 August 1991, where the AMPR polarization mixing produces $10 \mathrm{GHz}$ $T_{B}$ ranging from $95 \mathrm{~K}$ at scan start ( $\mathrm{H}$ polarization) to 147 $\mathrm{K}$ at scan finish ( $\mathrm{V}$ polarization). The dotted lines represent (1) applied to a radiative transfer model for various relative humidity profiles below $4-\mathrm{km}$ altitude. For a $50 \%$ relative humidity profile, the modeled $T_{B}$ agrees to within $2 \mathrm{~K}$ across the entire scan. 


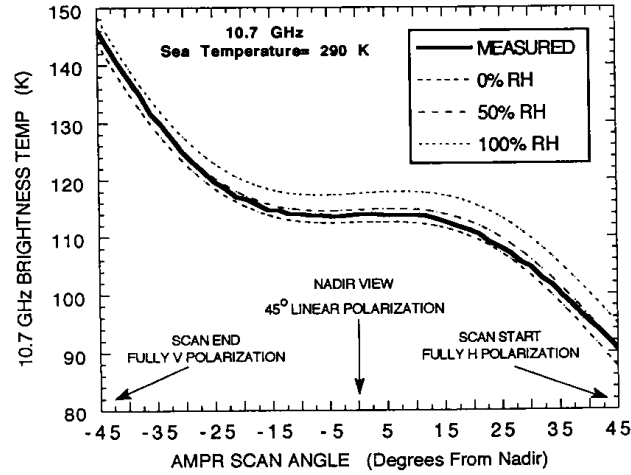

Fig. 1. An AMPR scan across a cloud-free ocean surface for the $10.7-\mathrm{GHz}$ channel from 12 August 1991. Radiative transfer model results are shown in dotted lines. The sea temperature is set to $290 \mathrm{~K}$, and the relative humidity profiles are constant below $4 \mathrm{~km} \mathrm{AGL}$, tapering to zero at $8 \mathrm{~km}$.

The additional orthogonal feedhorn ports on the MFFH are currently terminated; however, electronic techniques have been proposed which would allow the AMPR to be expanded into a radiometer with full dual-orthogonal linear polarization capability [28].

\section{AMPR Imagery from CaPE}

The identification of geophysical features is a nontrivial process in AMPR image analysis. With the knowledge of any fixed feature such as a lake or ocean boundary, any navigation drift in the ER-2 can be identified and corrected. The receive polarization state of AMPR changes across-track, and any polarimetric modeling of an AMPR scene should take into account this polarization mixing.

Emissivity of the terrestrial surface depends on the absorption and reflection properties of the material and its degree of roughness in relation to the wavelength. Between 10-100 $\mathrm{GHz}$, land surfaces have emissivities ranging between $0.6-0.9$, decreasing with soil moisture content. In general, microwave emissivity of water and wet soil surfaces increases with the frequency [29], unlike soil and vegetation surfaces which remain relatively frequency-independent. Fig. 2 shows a 4 panel AMPR scene taken during 2149-2155 UTC (about 80-km distance along-track) on 12 August 1991 during CaPE. The ER-2 flight bearing was $79^{\circ}$ which took it from land out over the Atlantic Ocean after 2153, about $100 \mathrm{~km}$ south of the CP-2 radar near the city of Melbourne. Over the ocean, the AMPR polarization mixing is evident at 10 and $19 \mathrm{GHz}$ as the reflector scans from the pilot's right ( $\mathrm{H}$ polarization) to the left ( $V$ polarization), gradually warming the $T_{B}$ in regions of little or no precipitation over the ocean (near 2155). This ocean emission signal is strongly depolarized at $85 \mathrm{GHz}$ due to strong emission from water vapor in the humid Florida environment, and effectively hides the ocean/land boundary at this frequency. This demonstrates the sensitivity of the $85 \mathrm{GHz}$ channel to water vapor; sample radiative transfer computations demonstrate that an $85 \mathrm{GHz} T_{B}$ of $260 \mathrm{~K}$ is easily achievable in a humid atmosphere with little or no columnar cloud water contents, regardless of the underlying surface.
Near a land-ocean boundary, the underlying emissivity changes abruptly, and some smearing (aliasing) is visible within the larger sized 10 and $19 \mathrm{GHz}$ footprints $(2.8-\mathrm{km}$ nadir resolution). Aliasing occurs when adjacent footprints each overlap each other. When viewing both portions of warm land and cold ocean a smearing effect is produced across adjacent footprints. Land $T_{B}$ ranges from $255-290 \mathrm{~K}$ with changes in the soil moisture, with adjacent lakes visible from $215030-215130$ in the $10-\mathrm{GHz}$ image $(180 \mathrm{~K})$, gradually becoming obscured at $37 \mathrm{GHz}$ and above due to poor $T_{B}$ contrast against the humid environment. It should be noted that although the aliasing effect is greatly minimized with the finescale resolution of all AMPR channels, it presents a problem for much coarser resolution spaceborne instruments such as the SSM/l. Since $T_{B}^{V} \geq T_{B}^{H}$ over the ocean, $T_{B}^{V}$ provides the least variability against a radiometrically warm land surface. The $\mathrm{V}$ polarized $T_{B}$ from the $\mathrm{SSM} / \mathrm{I}$ is often used when combining its channels for precipitation algorithms [30].

While $T_{B}$ increases with frequency for precipitation-free lakes and oceans, precipitation over land shows a decreasing trend in $T_{B}$ as the frequency increases. For ocean precipitation, $T_{B}$ initially increases with frequency, then decreases beyond a transition frequency. The transition frequency is highly dependent upon the strength of scattering processes within the cloud. At $85 \mathrm{GHz}$, three regions of convective activity are evident, two over land between $214930-215130$, and one along the coastline near 215330 . The cool $T_{B}$ regions surrounding the cores are stretched in the across-track dimension at 37 and $85 \mathrm{GHz}$, since the $T_{B}$ is responding to features in the upper ice region. This includes anvil regions, which cover a wider extent of the AMPR's across-track swath width. From 10 to $37 \mathrm{GHz}$, the rain region over the ocean near 215330 produces a $T_{B}$ warming, saturating near $260 \mathrm{~K}$. For the same region, the 37 and $85 \mathrm{GHz}$ images bear little resemblance. The 37 $\mathrm{GHz} T_{B}$ is ambiguous, since the $T_{B}$ over rain-free ocean is comparable to that produced by scattering effects over the ice canopies $(150-160 \mathrm{~K})$. This imagery illustrates the necessity of a multichannel configuration for reducing ambiguities in feature identification.

It is interesting to note that over land, the $10-\mathrm{GHz}$ AMPR $T_{B}$ is sensitive to regions of wet soil, swamps, and surface water. It is transparent to all but the strongest precipitation, and may be helpful in retrieving surface emissivity characteristics [17]. By comparing successive $10-\mathrm{GHz}$ images as a function of time, one could potentially gauge the accumulated rainfall for hydrological purposes. Over the ocean, the 10-GHz channel does not appear to saturate with increasing rain rate as quickly as the $19-\mathrm{GHz}$ channel.

\section{AMPR AND RADAR OBSERVATIONS OF PRECIPITATION}

We now focus our attention on analysis of the AMPR imagery over regions of precipitation. As shown in Fig. 2 , fine-scale $T_{B}$ features manifest themselves over regions surrounding the core of precipitation, especially at $85 \mathrm{GHz}$. To investigate this further, we have employed the use of the CP-2 radar products to compare the horizontal and vertical structure of precipitating clouds against the AMPR passive microwave 


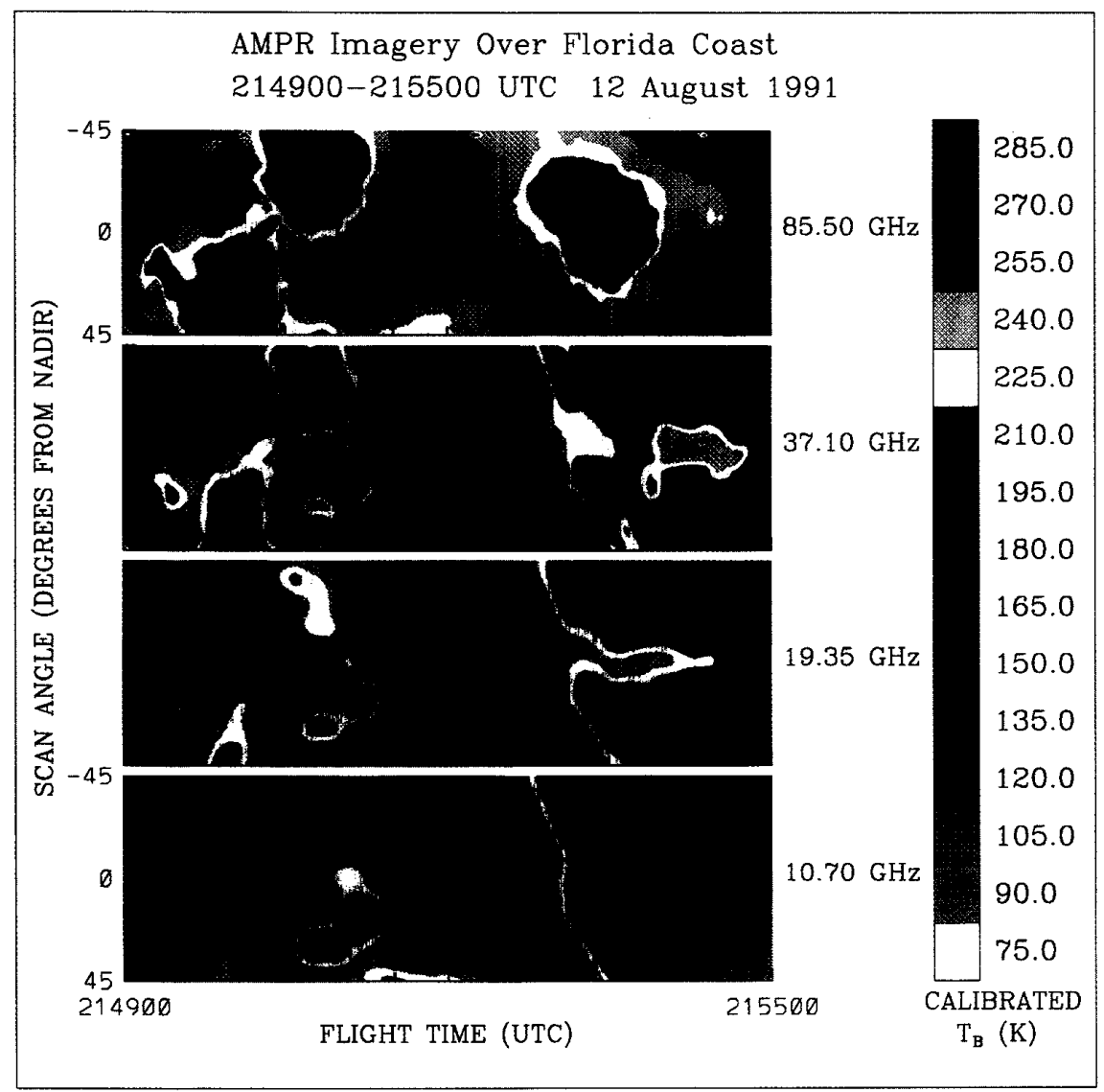

Fig. 2. AMPR imagery from $214900-215500$ UTC on 12 August 1991 . The ER-2 flight bearing was $79^{\circ}$ heading out over the Atlantic Ocean (right one third of figure). Three distinct regions of precipitation are visible in the $85-\mathrm{GHz}$ image. Two adjacent lakes are noted in the $10.7-\mathrm{GHz}$ image, with surrounding regions of wet soil (see text).

imagery. The four panels in Fig. 3 illustrate the AMPR imagery from 12 August 1991 during a 222615-222830 UTC pass (about 30-km distance along-track) of the ER-2 above a region of fairly strong precipitation near Daytona Beach, FL. The flight bearing was $2^{\circ}$ (nearly $\mathrm{S}$ to $\mathrm{N}$ ) along the Atlantic Ocean coastline. The center of each panel is located about 40 $\mathrm{km} \mathrm{NW}$ of the CP-2 radar, which itself was located just north of Merritt Island. The location and dimension of this storm cell place it over both land and ocean background, a feature which is easily noted in the $10 \mathrm{GHz} T_{B}$ imagery.

As discussed in Section II, the nadir AMPR polarization state is equally mixed ( $45^{\circ}$ linear polarization state) and idealized specular $\mathrm{H}$ and $\mathrm{V}$ ocean emissivities are equal. As a result, at $10 \mathrm{GHz}$, where the effects of emissive water vapor and oxygen are slight, the measured AMPR $T_{B}$ is coldest at the scan start $(90 \mathrm{~K})$ and quickly warms as the scan passes over land $(265-290 \mathrm{~K})$, with obvious aliasing effects occurring at 10 and $19 \mathrm{GHz}$. On top of this, the fraction of precipitation lying over the ocean manifests itself through a $T_{B}$ warming over the radiometrically cool ocean, although the exact amount of warming is difficult to determine due to variations in the underlying emissivity. Over land, there is a slight bulge in the
$10 \mathrm{GHz} 235 \mathrm{~K}$ contour (orange color) near the image center, which is likely caused by some emission warming and not a coastline feature.

At $19 \mathrm{GHz}$, more precipitation-sized ice and rain particles enter the Mie scattering regime, and the resultant scatteringinduced $T_{B}$ depressions are evident over the land region (Fig. 3 , upper right). Over land, a main core of $190 \mathrm{~K}$ is evident, as well as a slight $T_{B}$ depression near 222645 UTC, between the $+5^{\circ}$ and $-5^{\circ}$ scan region. The $19 \mathrm{GHz} T_{B}$ has warmed to $235 \mathrm{~K}$ over the portion of the storm over ocean, between the $20^{\circ}$ and $0^{\circ}$ scan region. In the lower right of the $19-\mathrm{GHz}$ image, a band of $160 \mathrm{~K}$ contour extends out over ocean. In general, over the portion of the precipitating cloud over ocean, the $T_{B}$ has warmed in relation to the $10-\mathrm{GHz}$ channel.

At 37 and $85 \mathrm{GHz}$, the most dominating feature is the wide extent of the $T_{B}$ depressions surrounding the main core (especially at $85 \mathrm{GHz}$ ), as these frequencies respond to processes higher in the cloud. Over ocean, the $37 \mathrm{GHz}$ $T_{B}$ has cooled in comparison to the $19 \mathrm{GHz}$ image as ice scattering begins to dominate emission warming. As in Fig. 2, a break in the $37-\mathrm{GHz}$ contour near $175 \mathrm{~K}$ occurs when the $T_{B}$ depression over land is about equal to the 


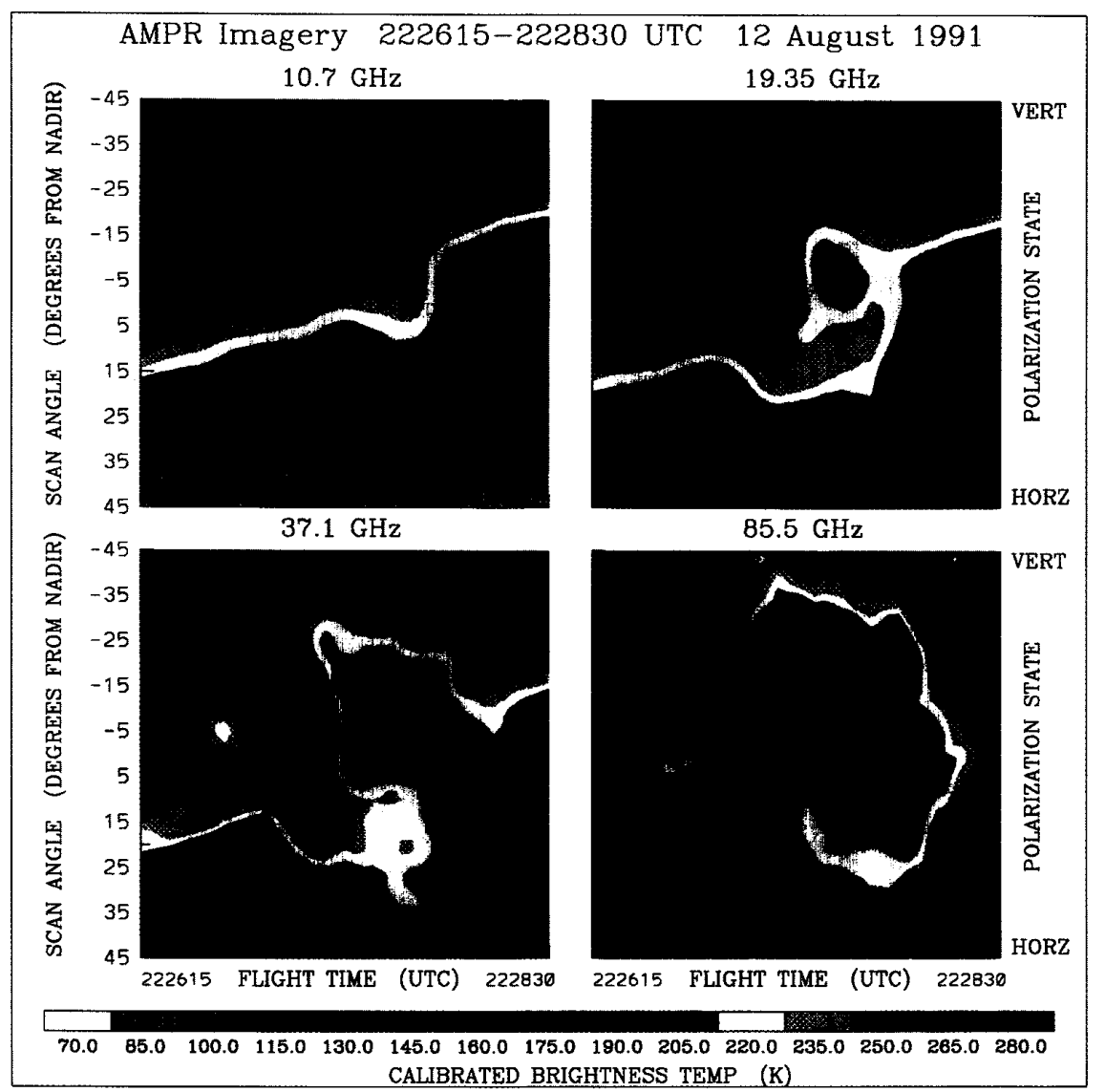

Fig. 3. AMPR imagery from $222615-222830$ UTC on 12 August 1991 . The ER- 2 flight bearing was $2^{\circ}$ heading along the Atlantic Ocean coastline. Two distinct regions of precipitation are visible in the $85-\mathrm{GHz}$ image (see text).

$T_{B}$ warming over ocean. Water vapor emission/absorption processes nearly obscure the ocean coastline at $85 \mathrm{GHz}$, and the $T_{B}$ over nonprecipitating land regions remains near $280 \mathrm{~K}$ at all frequencies. The smaller core near 222645 UTC becomes increasingly noticeable, producing $220 \mathrm{~K}$ and $175 \mathrm{~K}$ at 37 and $85 \mathrm{GHz}$, respectively, which are likely caused by somewhat less pronounced scattering mechanisms than those existing in the main core. The top portion of the $85 \mathrm{GHz}$ image from $-25^{\circ}$ to $-45^{\circ}$ scan angle, while producing $85 \mathrm{GHz} T_{B}$ below $200 \mathrm{~K}$ in some regions, actually views through the overlying ice anvil region, where underlying precipitation is nonexistent. In fact, there is quite a large extent of $85 \mathrm{GHz} T_{B}$ below $200 \mathrm{~K}$ in this image, but comparisons with the radar-derived rainfall display wide variability. Therefore, to provide insight into what precipitation processes are responsible for particular AMPR $T_{B}$ features, the CP-2 radar data was remapped into a format which accommodates the scanning operation of the AMPR radiometer system.

\section{A. AMPR-Viewed Remapping of Radar Products}

In order to accurately compare the collected radar data against the AMPR imagery, it becomes necessary to consider the regions within the cloud where particular $T_{B}$ features originate. In doing so, one invariably needs to consider how the cloud is structured, both vertically and horizontally, in terms of its microphysics-rain, graupel, cloud water, etc.-within the constantly changing FOV of the AMPR system. We present a scheme which facilitates the comparison between a radar PPI scanning mode and the AMPR scanning mode.

The CP-2 radar beams were first corrected for attenuation of the $X$ band copolar return [2], and the entire volume scan was remapped into a Cartesian coordinate system with the radar at the origin. The grid spacing was set to $0.5 \mathrm{~km}$ up to $15 \mathrm{~km}$, which retains the scale of the radar data as originally obtained in the PPI scan mode. The ER-2, however, flies at a $20-\mathrm{km}$ altitude above this coordinate system at a fixed flight bearing, and furthermore the emergent $T_{B}$ responds to different regions in and outside of the cloud as the AMPR scans across-track. This makes comparisons between conventional radar constantaltitude PPI's (CAPPI) and the AMPR imagery somewhat misleading. For example, if one of the AMPR channels (say 10 $\mathrm{GHz}$ ) was responding to a constant feature at a $2-\mathrm{km}$ altitude, the equivalent across-track scan would cover a distance of $2 \times$ $(20-2) \times \tan \left(45^{\circ}\right)=36 \mathrm{~km}$. If another channel (say $85 \mathrm{GHz}$ ) was responding to another feature at an $8 \mathrm{~km}$ altitude, the equivalent across-track distance at this height would be $2 \times$ 


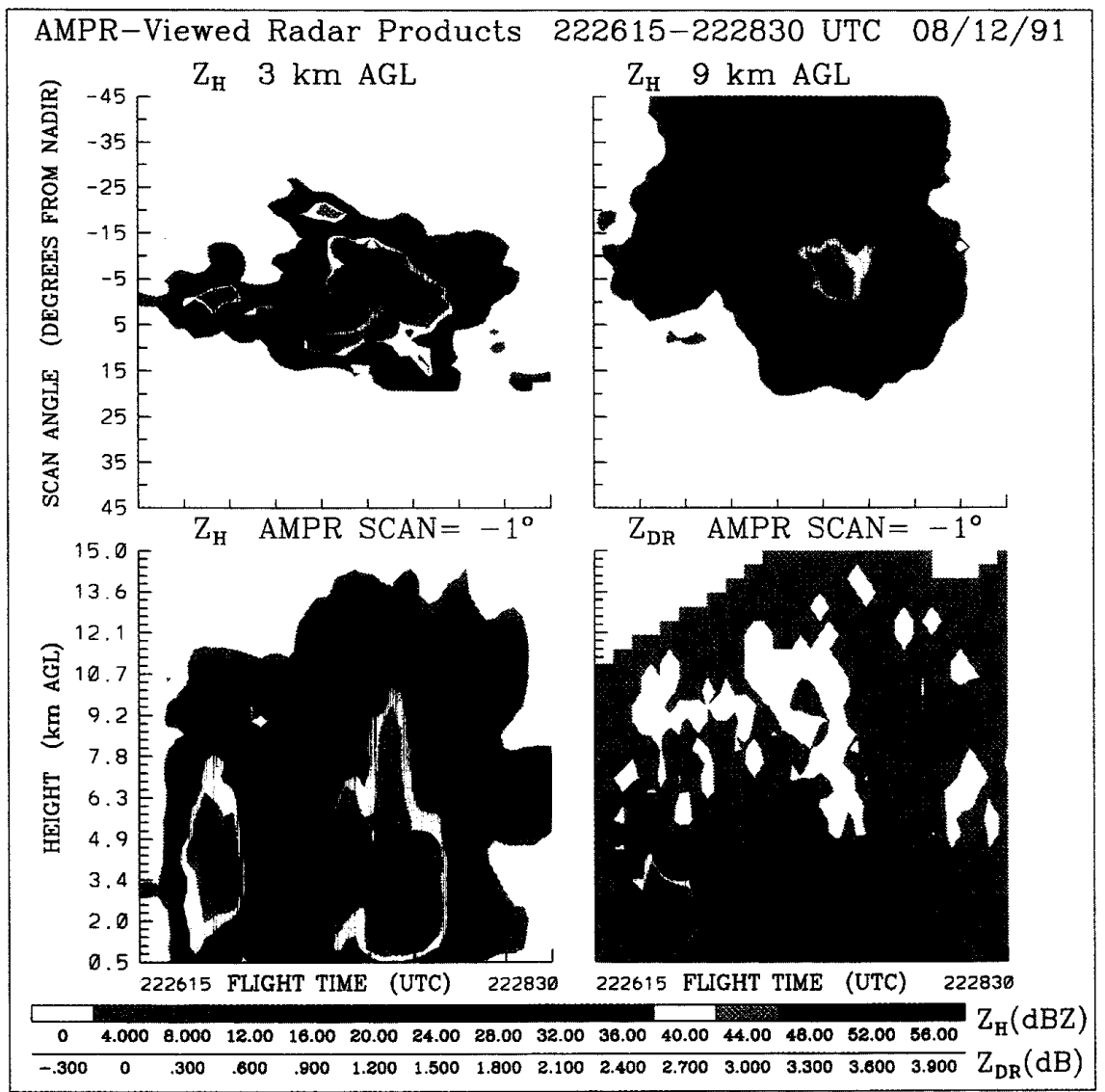

Fig. 4. CP-2 radar imagery from 222730-223030 UTC on 12 August 1991, remapped to compare against the AMPR imagery as the ER-2 flies above the cloud (see text). The $X$-axis limits correspond to the ER-2 flight segment from 222615 to $222830 \mathrm{UTC}$. (Top left) $3 \mathrm{GHz} Z$ in $\mathrm{dBZ}$ at a height of $3 \mathrm{~km}$ AGL. (Top right) $3 \mathrm{GHz} Z$ in dBZ at a height of $9 \mathrm{~km}$ AGL. (Lower left) Vertical profile of $3 \mathrm{GHz} Z$ in $\mathrm{dBZ}$ underneath the-1 ${ }^{\circ}$ scan angle. (Lower right) Vertical profile of $3 \mathrm{GHz} Z_{D R}$ in decibels underneath the $-1^{\circ}$ scan angle.

$(20-8) \times \tan \left(45^{\circ}\right)=24 \mathrm{~km}$. Given the $(x, y, z)$ coordinates of the ER-2 as a function of flight time, the AMPR scan angle, and the $(x, y, z)$ locations of the radar grid points, it is a simple geometric operation to extract the profiles of the radar data underlying each AMPR image pixel. In this manner the AMPR and radar images can be compared in either the along-track, across-track, or constant-altitude directions. Fig. 4 (upper left) illustrates the $S$ band $Z$ for a height of $3 \mathrm{~km}$ above ground level (AGL) from a CP-2 volume scan taken during 222730-223030 UTC (during the flight time of Fig. 3). Past $\approx 20^{\circ}$ scan angle, the CP- 2 radar did not gather data. Intuitively, with a series of these radar images at each height level, one should be able to identify the heights which best correspond to $T_{B}$ features in the AMPR image, since this is essentially the reflectivity that would be associated with a radar colocated and scanning in synchronization with the AMPR. In this case, $19 \mathrm{GHz} T_{B}$ features are spatially correlated with the $3 \mathrm{~km}$ height $Z$. The bulges in the $265 \mathrm{~K}$ contour toward the top of the image, the main core, and the $T_{B}$ warming over ocean - all three of these areas contain corresponding features in the $3 \mathrm{~km} Z$ image. The CP-2 scan limits just catch a portion of another region near 222830 and $15^{\circ}$ scan angle, which may be associated with the corresponding $T_{B}$ warming seen in the 19 and $37 \mathrm{GHz}$ images. At $19 \mathrm{GHz}$, it is difficult to remark quantitatively, since background effects are clearly obvious. It is safer to comment on the relative change between the 19 and $10 \mathrm{GHz} T_{B}$ images in relation to the observed reflectivity.

As the height of these remapped $Z$ images is raised, its correlation with the 19-GHz image gradually fades, suggesting that the AMPR/CP-2 correlation as a function of height is, in effect, a measure of the generalized weighting function proposed by Mugnai et al. [14], [25]. However, at the same time the correlation with the $37-\mathrm{GHz}$ images begins to increase, peaking near $5-6 \mathrm{~km}$ height (not shown), indicating that the $37 \mathrm{GHz}$ passive $T_{B}$ is a good measure of the complex region in and near the $0^{\circ} \mathrm{C}$ isotherm level, and is sensitive not only to rain and ice, but also the melting process. The most striking resemblance occurs for the $85-\mathrm{GHz}$ image. Fig. 4 (upper right) shows the remapped $Z$ at a $9 \mathrm{~km}$ height, where the overlying ice is clearly visible as a widespread 
region of $Z \approx 20 \mathrm{dBZ}$. The upper structure of the two cores is also clearly visible at 222630 UTC, and especially near 222730 , where $48 \mathrm{dBZ}$ reflectivity is noted. The resemblance between regions of $Z \geq 15 \mathrm{dBZ}$ and the $85-\mathrm{GHz}$ image (Fig. 3) visually demonstrates the effectiveness of using the 85 $\mathrm{GHz}$ channel for probing the upper cloud reaches. $85 \mathrm{GHz}$ $T_{B}$ of $115 \mathrm{~K}$ is noted near nadir over the main core, with the smaller, secondary core near 222630 UTC exhibiting a 180 $\mathrm{K}$ minimum. The vertical $Z$ and $Z_{D R}$ profiles (i.e., height versus ER-2 flight time) underneath the $-1^{\circ}$ scan (acrosstrack pixel 26 of 50) are shown in the lower left and lower right of Fig. 4, respectively (radar data was not used below $0.5 \mathrm{~km}$ height). Strong vertical motion within the main core has pushed the $40 \mathrm{dBZ}$ contour level up to $13 \mathrm{~km} \mathrm{AGL}$, with a colocated region of slightly negative $Z_{D R}$. Simulations of $Z_{D R}$ from hail [7] have shown that values of $\approx-1 \mathrm{~dB}$ are possible for oblate-shaped hail particles with their major axis oriented vertically. However, below $5 \mathrm{~km} \mathrm{AGL}$, the $Z_{D R}$ values in the main core are depressed with respect to those in the earlier, secondary core. $Z_{D R}$ values of near $3.5 \mathrm{~dB}$ are noted up to $4 \mathrm{~km}$ AGL in the secondary cell, while although positive $Z_{D R}$ extends up to $6 \mathrm{~km}$ in the main core, they reach only maximum values of near $2 \mathrm{~dB}$. Such conditions in this region (high $Z$, depressed $Z_{D R}$ ), are likely due to a rain/hail mixture [10], where a collection of aligned raindrops (providing perhaps 3-4 $\mathrm{dB} Z_{D R}$ ) and randomly oriented hail within the radar resolution volume produces a reduced overall $Z_{D R}$, near $2 \mathrm{~dB}$. Elevated $Z$ columns such as that noted here in the secondary cell are often found in vigorously growing convective clouds, where vertical air motion suspends supercooled cloud drops and rain above the $0^{\circ} \mathrm{C}$ level long enough to grow via collision and initiate the glaciation phase [31].

\section{B. Behavior of the 37 and $85 \mathrm{GHz} A M P R T_{B}$}

The difference between the 37 and $85 \mathrm{GHz} T_{B}$, referred to as $\Delta T_{B}$, is shown in Fig. 5 (upper left). The behavior of $\Delta T_{B}$ has been discussed with regard to its use as a measure of the integrated ice water path (IWP) [20]. $\Delta T_{B}$ values past $70 \mathrm{~K}$ are noted over the top of the figure, and $55 \mathrm{~K}$ is noted over the top of the smaller secondary cell at 222630 UTC. However, over the main core, the trend in $\Delta T_{B}$ is toward zero, and in some cases negative (compare with Fig. 4 , the $9 \mathrm{~km}$ AGL $Z$ ). This feature was also noted during AMPR engineering test flights in 1990. Even with inaccuracies in the $T_{B}$ calibration at the cold end, this strange trend is definitely present. One possible explanation involves a mixture of cloud water extending above a region of melting ice, where the $37 \mathrm{GHz}$ channel still responds deep enough into the region of ice, but the scattering effect at $85 \mathrm{GHz}$ is masked by the coexisting cloud water and ice. Previous modeling efforts have demonstrated that past a $10 \%$ (by volume) melting of exponential distributions of ice, the particles behave radiometrically as if they were nearly completely melted [23]. Therefore, in this case, an $85 \mathrm{GHz} T_{B}$ minimum of $115 \mathrm{~K}$ (as noted here) would only be achievable if additional nonmelted ice existed above. At this point, we refer to the remapped $\mathrm{CP}-2$ radar observations under the $-1^{\circ}$ scan shown in Fig. 4 ( $S$ band $Z$ and $Z_{D R}$ ), and also in Fig. 5 (DFRHS, $X$ band LDR, and one-way $X$-band attenuation rate $A_{X}$ ). The profiles in Fig. 5 are thresholded on $30 \mathrm{dBZ}$ reflectivity. Over the main core, the DFR-HS shows a $3 \mathrm{~dB}$ column from 4-11 km AGL. The typically quoted value at which the DFR-HS overcomes statistical fluctuations and provides a meaningful indication of the presence of Mie scatterers differs by several decibels. Tuttle and Rinehart investigated the impact of mismatched antenna patterns upon the DFRHS and proposed that values $\geq 3 \mathrm{~dB}$ indicate the existence of hail particle diameters greater than $1 \mathrm{~cm} \mathrm{[2].} \mathrm{Jameson} \mathrm{and}$ Heymsfield [3] indicated a lower bound of 3-5 $\mathrm{dB}$ for reliable detection of hail, while Herzegh and Jameson [4] proposed that DFR-HS values below $6 \mathrm{~dB}$ likely represent false hail indications. In the secondary core, the DFR-HS displays a 4-5 dB column between $3-8 \mathrm{~km}$ AGL. Therefore the secondary core may contain a broader hail size spectra and maximum size greater than $1 \mathrm{~cm}$, but through a smaller vertical column. Below $7 \mathrm{~km}$, regions of wet growth or melting are likely in both cores, since the $L D R$ shows an enhancement to near -15 $\mathrm{dB}$ and colocated with the region of highest $Z$ [12]. Regions of rain or melting, as indicated by the $A_{X}$ signal, lie near 0.4 $\mathrm{dB} / \mathrm{km}^{-1}$ in an elevated region within the secondary core. In the main core, an attenuation bright band with peak values of $\approx 1.4 \mathrm{~dB} / \mathrm{km}^{-1}$ suggests the presence of small melting hail [32].

In sum, a likely scenario for the main core consists of strong vertical motion suspending some hail above $7 \mathrm{~km}$ AGL, with melting hail and possibly a rain/hail mixture existing below this. Based on earlier radar observations, the secondary cell is at an earlier stage of evolution, with wet growth occurring near and above the $0^{\circ} \mathrm{C}$ level, light rain fallout at the surface, and some evidence of glaciation at the $6 \mathrm{~km}$ level. With such strong upward vertical motion within the main core, it is possible that a dominant size sorting phenomenon is occurring. Such a dominant size could preferentially enhance the ice scattering at $37 \mathrm{GHz}$ and produce the unusually low $T_{B}$ noted over this core region.

To attempt to explain this $\Delta T_{B}$ discrepancy, we modeled various monodisperse distributions of high $\left(0.91 \mathrm{~g} \mathrm{~cm}^{-3}\right)$ and low $\left(0.45 \mathrm{~g} \mathrm{~cm}^{-3}\right)$ density ice spheres using a microwave radiative transfer model [20]. A monodisperse distribution was chosen to observe the particular range of radii over which any peculiar $T_{B}$ behavior may occur. A constant number concentration of $10 \mathrm{~m}^{-3}$ was used independent of the radius of the sphere. The model consisted of a 4-km ice layer placed above a $4 \mathrm{~km}, 75 \mathrm{~mm} / \mathrm{h}^{-1}$ rain layer. The associated $S$ and $X$ band equivalent radar reflectivities $Z_{e q}$ in the ice region were also calculated. Fig. 6 shows a plot of the upwelling 10 19,37 , and $85 \mathrm{GHz} T_{B}$ at $8^{\circ}$ from nadir as a function of ice sphere radius. For high density ice with a radius between 3-4 mm, the $37 \mathrm{GHz} T_{B}$ can actually fall below that of 85 $\mathrm{GHz}$ by up to $30 \mathrm{~K}$, with an associated $S$ band $Z_{e q} \approx 45-50$ $\mathrm{dBZ}$ and corresponding DFR-HS values below $2 \mathrm{~dB}$. The variations in the $T_{B}$ curves are due to the oscillatory nature of the normalized Mie extinction cross section as size parameter $\chi=2 \pi r / \lambda$ increases. Since the dielectric constant of ice is only weakly dependent upon frequency and temperature, 


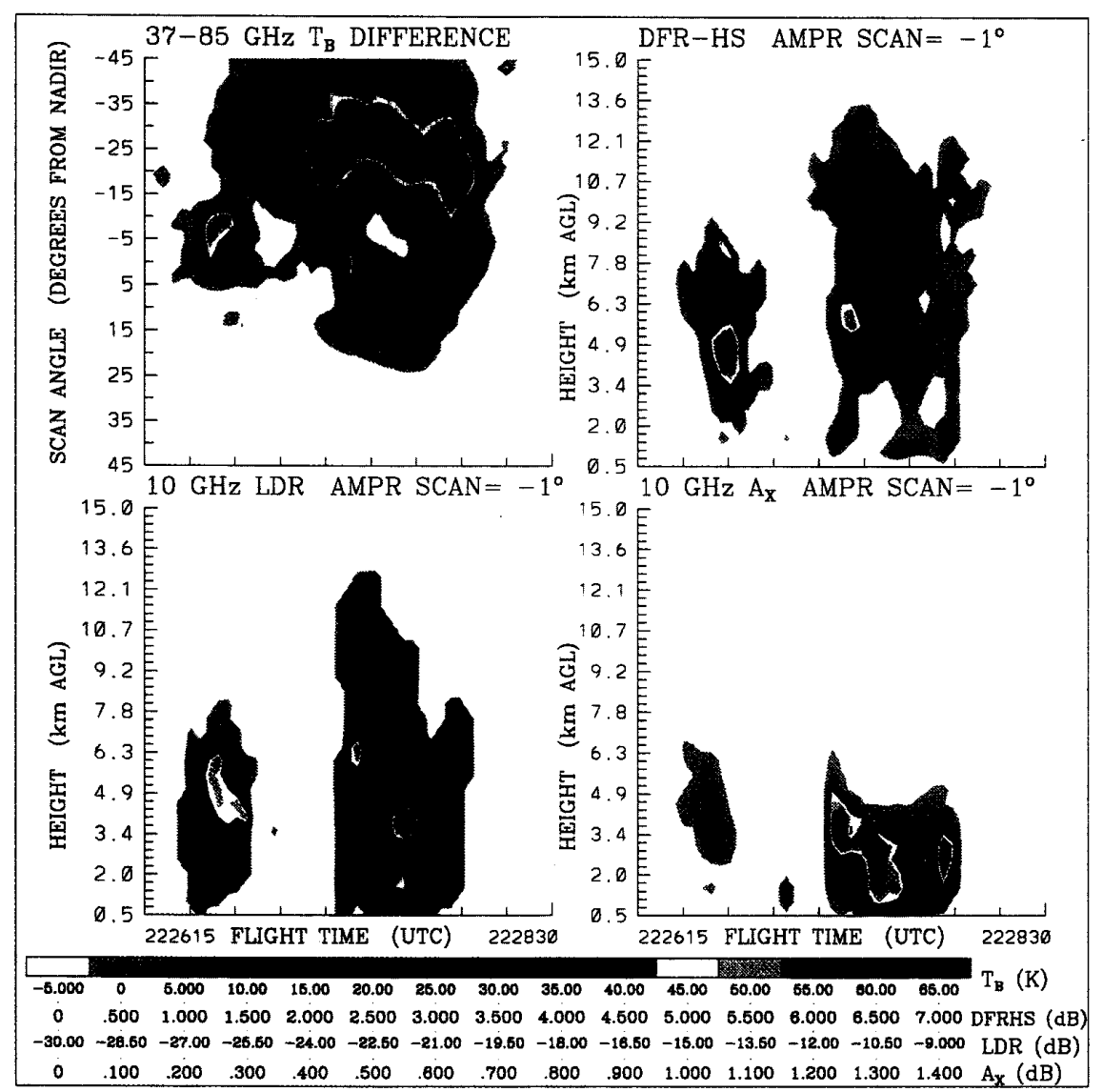

Fig. 5. CP-2 radar imagery from 222730-223030 UTC on 12 August 1991, remapped to compare against the AMPR imagery as the ER-2 flies above the cloud (see text). The $X$-axis limits correspond to the ER-2 flight segment from 222615 to 222830 UTC. (Top left) AMPR $37-85$ GHz $T_{B}$ difference. (Top right) Dual frequency ratio hail signal (DFR-HS) in decibels underneath the $-1^{\circ}$ scan angle. (Lower left) Vertical profile of $10 \mathrm{GHz} L D R$ in decibels underneath the $-1^{\circ}$ scan angle. (Lower right) Vertical profile of $10-\mathrm{GHz}$ one-way attenuation rate $A_{X}$ in decibels/kilometer ${ }^{-1}$ underneath the $-1^{\circ}$ scan angle.

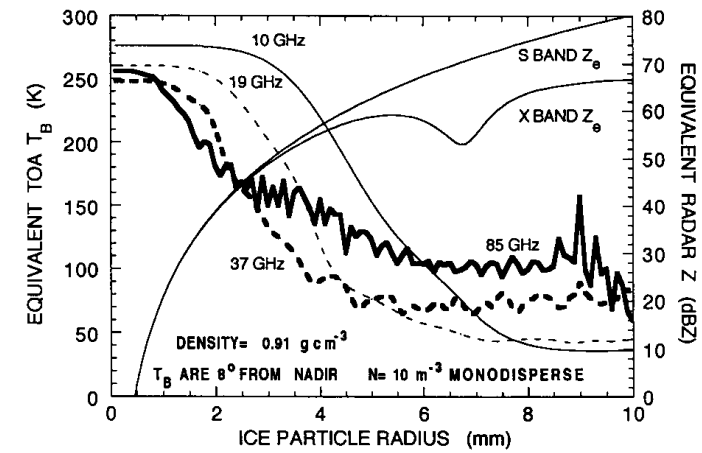

Fig. 6. Model-simulated upwelling $10,19,37$, and $85 \mathrm{GHz} T_{B}$ at an angle of $8^{\circ}$ from nadir as a function of ice sphere radius. The radiative transfer model consists of a $4-\mathrm{km}$ layer of ice overlying a $4-\mathrm{km}, 75 \mathrm{~mm} / \mathrm{h}^{-1}$ rain layer. The ice layer is characterized by a monodisperse distribution with a number concentration of $10 \mathrm{~m}^{-3}$ and a density of $0.91 \mathrm{~g} \mathrm{~cm}^{-3}$. The associated $S$ and $X$ band ice equivalent radar reflectivities $Z_{e q}$ are also shown.

a given particle radius enters the resonance region at a successively smaller radius as frequency increases. As a result, the $85 \mathrm{GHz}$ scattering cross section can be smaller than that at $37 \mathrm{GHz}$ over a limited range of radii. In this case, these modeled $Z_{e q}$ values approximate the CP-2 $S$ band $Z$ and weak DFR-HS values observed in the upper ice region of the main core.

Many microwave radiative transfer models designed for precipitation retrieval use exponential distributions for modeling rain and ice particles. While such distributions may effectively simulate stratiform rain conditions and regions where vertical upward motion is reduced, in strong convective cores the choice of a suitable ice distribution is highly variable. Both radar and AMPR observations are consistent with radiative transfer model output which supports the suggestion that a dominant ice size is present in high reflectivity ice columns supported by strong upward motion, such as a sharply dispersed gamma size distribution. However, the above model is simplistic in the fact that polarization effects have not been considered, nor aspherical ice particles. We offer this as one possible explanation for the negative $\Delta T_{B}$ observed by the AMPR over the cores of strong convective storms, and may be a viable passive microwave remote measure of the "convective strength" of a storm (i.e., of the magnitude of the upward vertical velocity). 


\section{CONCLUSIONS AND CURRENT EFFORTS}

The complementary nature of combined active and passive observations of precipitating clouds has been demonstrated. While passive measurements (either ground or space based) represent integrated effects from emission, absorption, and scattering within the radiometer's field of view, active radar measurements probe a specific location within the cloud including attenuation along the beam. The radiative effects manifested at such a high resolution scale provide more detailed information on the complex microphysical structure of precipitation. While such high resolution measurements are not possible from current satellite altitudes, the TRMM satellite will provide radiometer resolutions ranging from 37 $\mathrm{km}$ at $10 \mathrm{GHz}$ to $5 \mathrm{~km}$ at $90 \mathrm{GHz}$. Comparisons of radar and AMPR images have illustrated that the $85 \mathrm{GHz} T_{B}$ is inversely related to the radar reflectivity at heights of $9-10$ $\mathrm{km}$. Nineteen gigahertz $T_{B}$ depressions and increases against a land and ocean background, respectively, show a natural correlation with the reflectivity near $3 \mathrm{~km}$ height. Over land, a $37 \mathrm{GHz}$ channel appears to sense the microphysically complex region near $0^{\circ} \mathrm{C}$. Over the ocean, the lowest AMPR channel of $10 \mathrm{GHz}$ showed continued $T_{B}$ warming from rainfall after the $19-\mathrm{GHz}$ channel had saturated near $260 \mathrm{~K}$. While initial tendencies are to dismiss the applicability of the 10 $\mathrm{GHz}$ channel for land precipitation, the imagery shown in Fig. 2 shows that measurable $T_{B}$ depressions occur with soil wetting, or possibly from extreme $10-\mathrm{GHz}$ ice scattering effects. This gives an indication of the surface emissivity and also the evolutionary state of the storm. Over the main storm core, the $37-85 \mathrm{GHz} T_{B}$ difference $\Delta T_{B}$ approached and in some regions fell below zero. Radiative transfer model results indicate that the presence of dominant ice sizes near 3-4 mm radius was consistent with both CP-2 and AMPR observations above $7 \mathrm{~km}$ height. This unique signature $\left(85 \mathrm{GHz} T_{B} \leq\right.$ $150 \mathrm{~K}$ and $\Delta T_{B} \rightarrow 0$ ) could potentially be used as a means to gauge a storm's "convective strength," since in this and other AMPR imagery it appeared to be associated with strong upward vertical velocities and an extended reflectivity column.

The main difficulty associated with using radar as verification for satellite-based microwave precipitation retrievals derives from inherent ambiguities associated with inverting the radar measurements into physical cloud quantities, such as size distributions and water/ice fractions. While the comparisons presented here have necessarily been of a qualitative nature, a microwave radiative transfer model provides the necessary link between the inverted radar products and the observed AMPR $T_{B}$. We are testing the effectiveness of our radar inversion techniques by comparing radiative transfer model $T_{B}$ output against the AMPR $T_{B}$ imagery. Based on this and previous modeling studies, we can state with ample evidence that there are two key deficiencies in the radar modeling [20]. The first and foremost of these lies with derivation of the ice size distribution and its associated density, both of which display wide variabilities depending upon the cloud evolutionary state and the specific location within the cloud. The second involves locating and quantifying the regions of cloud water. Although $3-\mathrm{GHz}$ radar systems are relatively unaffected by propagation effects, cloud water is usually fairly well spread out within the cloud, and its associated radar echo is too weak to detect with any certainty below $10 \mathrm{GHz}$.

Similarly, when considering multispectral passive measurements from space, the inverse problem of deriving a vertical cloud structure from a finite set of multispectral passive measurements is by nature nonunique. One must resort to some additional information to narrow onto a realistic solution. One encouraging approach involves the incorporation of a cloud-mesoscale model with time-dependent microphysics to generate a large database of various hydrometeor profiles. A physically and statistically based algorithm then attempts to locate the best candidate hydrometeor profiles against a set of actual multispectral $T_{B}$ observations [17], [25]. We are currently combining additional CP-2 radar and radiative transfer modeling efforts from CaPE into this precipitation retrieval method, with both SSM/I and AMPR measurements providing the multispectral $T_{B}$ input. Quantitative results are very encouraging and we will be reporting on the final results in a future series of publications.

\section{ACKNOWLEDGMENT}

The authors thank J. Lutz and the NCAR radar staff for their efforts in transporting, installing, and maintaining the $\mathrm{CP}-2$ radar during CaPE. The CEDRIC radar format software developed by W. Anderson and L. J. Miller of NCAR/MMM facilitated the radar data analysis. R. Hood and V. L. Griffin of NASA/MSFC directed and coordinated the ER-2 flights during $\mathrm{CaPE}$, and $\mathrm{F}$. LaFontaine carefully preprocessed the AMPR datasets. The authors are grateful to Dr. R. Spencer of NASA/MSFC for his support of spaceborne passive microwave radiometric methods and instrumentation.

\section{REFERENCES}

[1] J. Simpson, R. F. Adler, and G. North, "A proposed tropical rainfall measuring mission (TRMM) satellite," Bull. Amer. Meteorol. Soc., vol. 69, pp. 278-295, 1988.

[2] J.D. Tuttle and R.E. Rinehart, "Attenuation correction in dualwavelength analyses," J. Clim. Appl. Meteor., vol. 22, pp. 1914-1921, 1983.

[3] A.R. Jameson and A.J. Heymsfield, "Hail growth mechanisms in a Colorado storm. Part I: Dual wavelength radar observations," J. Atmos. Sci., vol. 37, pp. 1763-1778.

[4] P.H. Herzegh and A.R. Jameson, "Observing precipitation through dualpolarization radar measurements," Bull. Amer. Meteorol. Soc., vol. 73, pp. 1365-1374, 1992.

[5] M. Sachidananda and D. S. Zrnic, "Differential propagation phase shift and rainfall rate estimation," Radio Sci., vol. 21, pp. 235-247, 1986.

[6] M. P. M. Hall, W. F. Goddard, and S. M. Cherry, "Identification of hydrometeors and other targets by dual-polarization radar," Radio Sci., vol. 19 , pp. $132-140,1984$.

[7] V. N. Bringi, J. Vivekanandan, and J. D. Tuttle, "Multiparameter radar studies in Colorado convective storms. Part 2: Hail detection studies," J. Atmos. Sci., vol. 43, pp. 2564-2577, 1986.

[8] A. R. Jameson, "Microphysical interpretation of multiparameter radar measurements in rain. Part 1: Interpretation of polarization measurements and estimation of raindrop shapes," J. Atmos. Sci., vol. 40, pp. 1792-1802, 1983.

[9] A.J. Illingworth and I.J. Caylor, "Cross polar observations of the bright band," in Proc. 24th AMS Conf. Radar Meteor., Tallahassee, FL, 1989, pp. 323-327.

[10] N. Balakrishnan and D.S. Zrnic, "Use of cross correlation between orthogonally polarized echoes to characterize precipitation and infer hail size," J. Atmos. Sci., vol. 47, pp.1525-1540, 1990. 
[11] N. Balakrishnan and D. S. Zrnic, "Estimation of rain and hail rates in mixed-phase precipitation," J. Atmos. Sci., vol. 47, pp. 565-583, 1990

[12] R. Fulton and G.M. Heymsfield, "Microphysical and radiative characteristics of convective clouds during COHMEX," J. Appl. Meteorol., vol. 30, pp. 98-116, 1991.

[13] R. W. Spencer, H.M. Goodman, and R.E. Hood, "Precipitation retrieval over land and ocean with the SSM/I: Identification and characteristics of the scattering signal," J. Atmos. Ocean. Tech. vol 6, pp. 254-273, 1989.

[14] A. Mugnai, H.J. Cooper, E.A. Smith, and G.J. Tripoli, "Simulation of microwave brightness temperatures of an evolving hailstorm at SSM/1 frequencies," Bull. Amer. Meteorol. Soc., vol. 71, pp. 2-13, 1990.

[15] C. Kummerow, I.M. Hakkarinen, H.F. Pierce, and J.A. Weinman, "Determination of precipitation profiles from airborne passive microwave radiometric measurements," J. Atmos. Ocean. Tech., vol. 8, pp. 148-158, 1991.

[16] A. Mugnai and E. A. Smith, "Radiative transfer to space through precipitating cloud at multiple microwave frequencies. Part 1: Model description," J. Appl. Meteorol., vol. 27, pp. 1055-1073, 1988.

[17] E. A. Smith, A. Mugnai, H. J. Cooper, G. Tripoli, and X. Xiang, "Foundations for statistical-physical precipitation retrieval from passive microwave satellite measurements. Part 1: Brightness-temperature properties of a time-dependent cloud-radiation model," J. Appl. Meteorol., vol. 31, pp. 506-531, 1992.

[18] T. T. Wilheit, M. S. Rao, E. B. Rodgers, and J. S. Theon, "A satellite technique for quantitatively mapping rainfall rates over the oceans," $J$. Appl. Meteorol., vol. 16, pp. 551-560, 1977.

[19] R. F. Adler, H. M. Yeh, N. Prasad, W. Tao, and J. Simpson, "Microwave simulations of a tropical rainfall system with a three-dimensional cloud model," J. Appl. Meteorol., vol. 30, pp. 924-953, 1991.

[20] J. Vivekanandan, J. Turk, and V. N. Bringi, "Ice water path estimation and characterization using passive microwave radiometry," J. Appl. Meteorol., vol. 30, pp. 1407-1421, 1991.

[21] E. A. Smith and A. Mugnai, "Radiative transfer to space through precipitating cloud at multiple microwave frequencies. Part 3: Influence of large ice particles," J. Meteorol. Soc. Jap., vol. 67, pp. 739-754, 1989.

[22] K.F. Evans and G. L. Stephens, "A new polarized atmospheric radiative transfer model," J. Quant. Spectrosc. Radiat. Transfer, vol. 46, pp. 413-413, 1991.

[23] J. Vivekanandan, J. Turk, G.L. Stephens, and V.N. Bringi, "Microwave radiative transfer studies using combined multiparameter radar and radiometer measurements during COHMEX," J. Appl. Meteorol., vol. 29, pp. 561-585, 1990.

[24] G. B. Foote, "Scientific overview and operations plan for the Convection and Precipitation/Electrification Experiment (CaPE)," National Center for Atmospheric Research Report, Boulder, CO, 1991.

[25] A. Mugnai, E.A. Smith, and G. Tripoli, "Foundations for statistical physical precipitation retrieval from passive microwave satellite measurements. Part 2: Emission source and generalized weighting function properties of a time-dependent cloud-radiation model," J. Appl. Meteorol., vol. 31 .

[26] V. N. Bringi and A. Hendry, "Technology of polarization diversity radars for meteorology," in Radar in Meteorology. Boston, MA: AMS, 1990, ch. $19 \mathrm{a}$, pp. $153-190$.

[27] J.A. Galliano, R.H. Platt, R.E. Hood, R.W. Spencer, V.L. Griffin M.W. James, and F.J. LaFontaine, "Advanced microwave precipitation radiometer observations during the Convection and Precipitation/ Electrification Experiment," in Proc. NOAA/WPL Specialists Meet. Microwave Radiometry and Remote Sensing Appl., Boulder, CO, Jan. 14-16, 1992, pp. 428-432.

[28] A.J. Gasiewski and D.B. Kunkee, "Laboratory demonstration of electronic polarization basis rotation," presented at the IEEE MTT-S Int Microwave Symposium, 1992.

[29] R. R. Ferraro, N. C. Grody, and J. A. Kogut, "Classification of geophysical parameters using passive microwave satellite measurements," IEEE Trans. Geosci. Remote Sensing, vol. 24, pp. 1008-1013, 1986.
[30] N.C. Grody, "Classification of snow cover and precipitation using the Special Sensor Microwave Imager," J. Geophys. Res., vol. 96, pp. 7423-7435, 1991.

[31] J. D. Tuttle, V. N. Bringi, H. D. Orville, and F. J. Kopp, "Multiparameter radar study of a microburst: Comparison with cloud model results," $J$. Atmos. Sci., vol, 46, pp. 601-620, 1989.

[32] J. Vivekanandan, V. N. Bringi, and R. Raghavan, "Multiparameter radar modeling and observation of melting ice," J. Atmos. Sci., vol. 47, pp. $549-564,1990$.

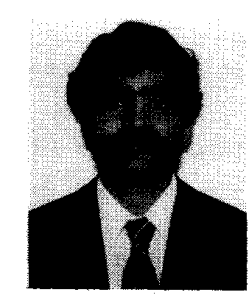

J. Vivekanandan received the B.E. degree in electronics and communication engineering from Madural-Kamaraj University, the M.Tech. degree in microwave and radar engineering from the Indian Institute of Technology, Kharagpur, India, and the $\mathrm{Ph} . \mathrm{D}$. degree in electrical engineering in 1986 from Colorado State University, Fort Collins

At present he is a scientist at the National Center for Atmospheric Research, Boulder, CO. His research experience includes microwave radar and radiometer/satellite remote sensing of the atmosphere, vegetation, and soil. Recently, he has spent considerable effort in modeling polarimetric radar and multifrequency radiometer observations of clouds and their interpretations.

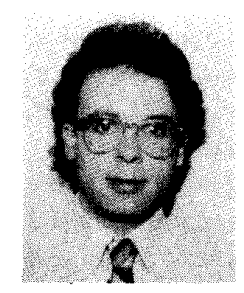

Joseph Turk (S'91-A'92) was born in Hibbing, $\mathrm{MN}$, on August 25, 1960. He received the B.S. and M.S. degrees in electrical engineering from Michigan Technological University in 1982 and 1984, and the Ph.D. degree from Colorado State University (CSU) in 1991.

During 1984-1986 he was with the Communications Group at Motorola, Inc. Schaumberg, IL, where he worked on the development of cellular mobile telephones. From 1986 to 1990 , he was an Army Center for Geosciences Fellow at CSU where his research focused upon passive microwave radiometry, microwave radiative transfer, and polarimetric radar applied to satellite remote sensing of precipitation. His additional research areas are particle scattering theory and atmospheric radio propagation. Currently he is involved with the NASA Advanced Communications Technology Satellite (ACTS) Ka-band propagation measurement program at $\mathrm{CSU}$.

V. N. Bringi was a member of the AMS Committee on Radar Meteorology. $\mathrm{He}$ is currently a member of the NEXRAD Technical Advisory Committee. His research interests over the last 15 years have been in the areas of electromagnetic wave propagation and scattering, multiple scattering of waves, polarimetric radar techniques, and applications to meteorology.

Dr. Bringi has received the following honors and awards: UCAR Fellowship (1974-1976), the Alumni Award for Graduate Student Research (1977) from Ohio State University, Colorado State University College of Engineering's Young Faculty Professional Development Award (1983), the Halliburton Young Faculty Research Award (1984), and the Dean's Council Award (1989). 